\title{
Les épisodes de poussières désertiques en France métropolitaine de début 2021
}

\author{
Michaël Kreitz' ${ }^{1}$ Gaétan Heymes ${ }^{2}$ \\ 1. École nationale de la météorologie, Météo-France, Toulouse \\ 2. Centre météorologique de Briançon, Météo-France, Briançon
}

\begin{abstract}
a fin d'hiver et le début de printemps météorologiques 2021 ont été marqués par de nombreux épisodes de remontées de poussières désertiques. Ces situations n'ont rien d'exceptionnel, mais leur répétition et leurs conséquences quelquefois spectaculaires méritent quelques précisions.
\end{abstract}

\section{Situations synoptiques}

Parmi les nombreux épisodes qui se sont succédé, quatre semblent plus intenses : les 5 et 6 février, 21 et 22 février, 2 et 3 mars, 2 et 3 avril. Les situations sont proches d'un point de vue synoptique (figure 1) avec un talweg du proche atlantique jusque vers le Maroc et des hautes valeurs de pression de surface entre la Méditerranée et l'Europe centrale. Ces centres d'action ainsi positionnés pilotent un flux de sud à sud-ouest à grande échelle venant transporter les poussières désertiques issues du Sahara jusqu'en Europe de l'Ouest. Parmi ces situations, les deux premières sont nettement plus perturbées, avec un flux plus rapide. Ces situations sont conformes avec l'étude de Di Mauro et al. (2019) selon laquelle, pour l'Europe de l'Ouest et plus particulièrement les Alpes, ce sont les bassins nord du Sahara et le Sahel (Tchad, Hoggar, bassin du Niger et nord Algérie) qui contribuent le plus souvent aux événements observés.

Selon l'InVS ${ }^{1}$, de manière plus générale, la majorité des poussières désertiques se dirige vers le golfe de Guinée, une plus petite partie vers l'Atlantique
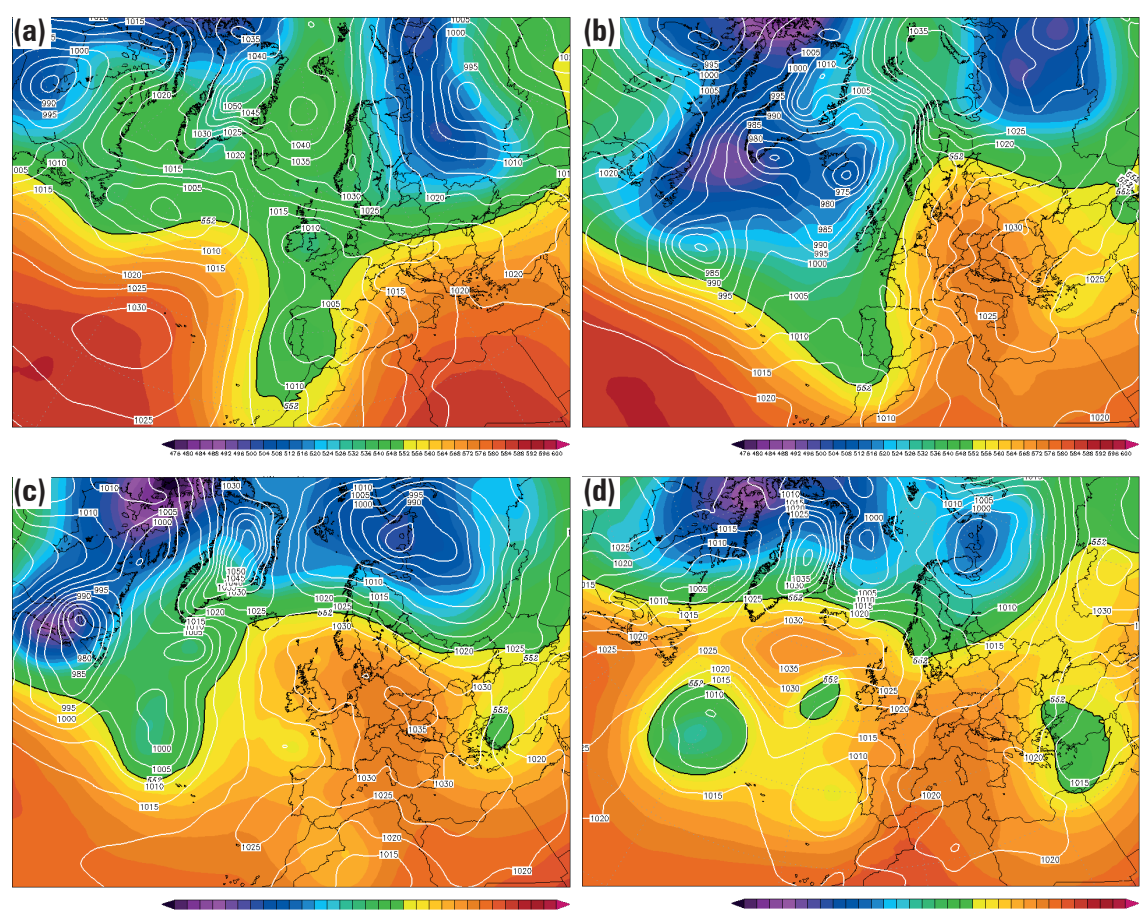

Figure 1. Pression réduite au niveau de la mer (Pmer, isolignes blanches) et géopotentiel à $500 \mathrm{hPa}$ (décamètre géopotentiel, damgp, couleurs) : de gauche à droite et de haut en bas, les 6 février à $0 \mathrm{~h}$ TU, 21 février à $12 \mathrm{~h}$ TU, 2 mars à $12 \mathrm{~h}$ TU et $1^{\text {er }}$ avril à $0 \mathrm{~h}$ TU. Source : Wetterzentrale.

(voir Cottereau, 2020) et une proportion plus réduite encore vers l'Europe. En Europe, ce sont les régions d'Europe du Sud et de l'Est qui sont les plus concernées et à un degré moindre l’Europe de l'Ouest. Les périodes d'avril à juin sont les plus propices aux poussières désertiques, alors qu'octobre à décembre est une période creuse. Plus précisément, selon Pey et al. (2013), les cas hivernaux correspondent, comme ici pour les trois premières situations, au courant perturbé qui arrive à transporter très rapidement des poussières en grandes quantités et sur des distances importantes, plutôt sur l'est du bassin méditerranéen. En été, l'ouest du bassin subit durablement la présence de poussières par des processus convectifs au-dessus du Sahara combinés à des hautes valeurs de pression plus au nord.

Les images satellitaires illustrent toutes la présence de poussières en suspension sous la forme d'un voile

1. Institut de veille sanitaire. Note de synthèse relative à la problématique des vents de sable en provenance des déserts, 2012. http://www. qualitaircorse.org/userfiles/files/Etude_InVS_ CIRE_vents_de_sable.pdf 

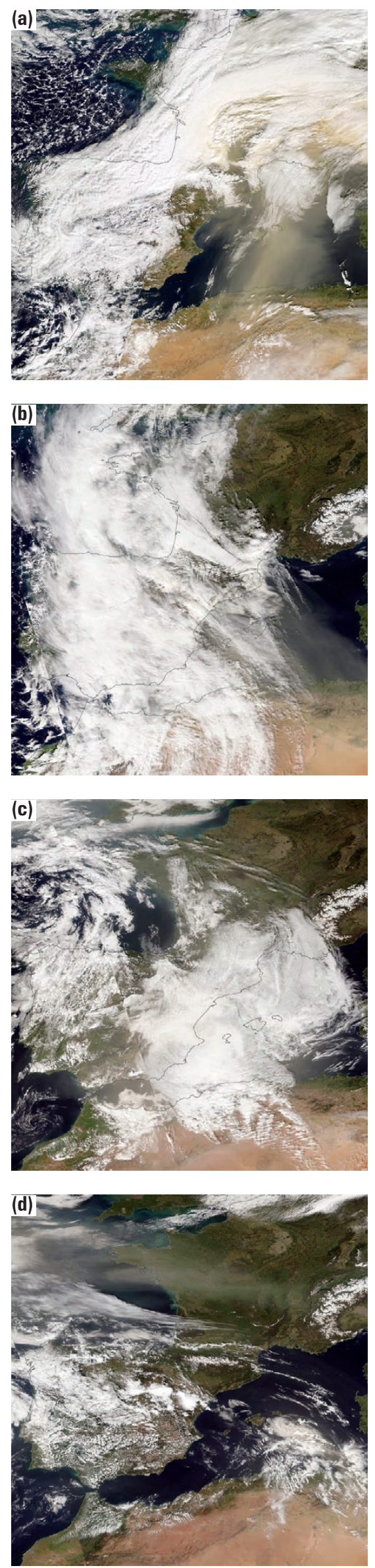

Figure 2. Images satellitaires en composition colorée, de gauche à droite et de haut en bas, les 6 février (Terra), 21 février (Aqua), 2 mars (Suomi NPP) et 2 avril (Suomi NPP). Source Nasa Worldview. jaunâtre (figure 2) entre le Maghreb et la France. La situation du 6 février semble la plus spectaculaire en termes de concentration de poussières, alors que celle du 2 avril est clairement associée à des conditions anticycloniques.

\section{Situation des 5 et 6 février 2021}

La situation des 5 et 6 février, déjà spectaculaire depuis le satellite, voit une ambiance jaunâtre quasi irréelle au lever du jour sur la moitié sud-est de la France (figure 5). Les dépôts de poussières sont conséquents, en particulier sur les Pyrénées et les Alpes, le plus souvent sans précipitations notables. Il s'agit pour beaucoup de dépôts secs. De nombreuses vidéos spectaculaires sont publiées sur les réseaux sociaux, donnant l'impression de faire du ski en plein milieu du Sahara... Sur la figure 3 , les poussières désertiques présentent en composition colorée une teinte jaunâtre. Eumetsat a également mis au point une composition colorée spécifique,qualifié de "CC dust ", identifiant les poussières désertiques en rose (figure 3). Les poussières sont présentes au-dessus de la Méditerranée et bien plus au nord certainement, mais les nuages empêchent la détection (même si la teinte rose est observée au-dessus du piémont pyrénéen, dans une petite poche d'éclaircies).

La veille, le satellite Terra permet de voir les poussières se faire arracher depuis le nord du Maghreb à la frontière entre Maroc et Algérie (figure 4).
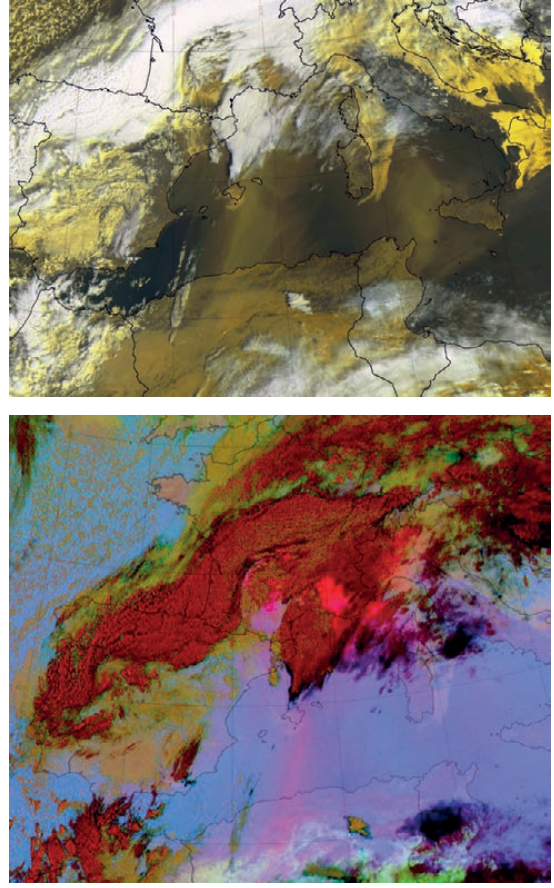

Figure 3. Comparaison entre la composition colorée (classique) de Metop-2 (en haut) et la composition colorée spécifique aux poussières de MSG-4 (en bas) le 6 février 2021 à 8 h 30 TU. Source : Météo-France.

Cet épisode aura donc laissé des quantités imposantes de poussières sur les neiges des Pyrénées et des Alpes (phénomène déjà évoqué dans ce numéro avec l'image du trimestre). Les jours suivants, ces poussières seront recouvertes par de la neige fraîche, donnant l'impression qu'elle a disparu. Pourtant, les sondages réalisés indiquent la persistance et l'homogénéité de la couche (figure 5).

Les poussières déposées sur le manteau neigeux en modifient l'albédo.

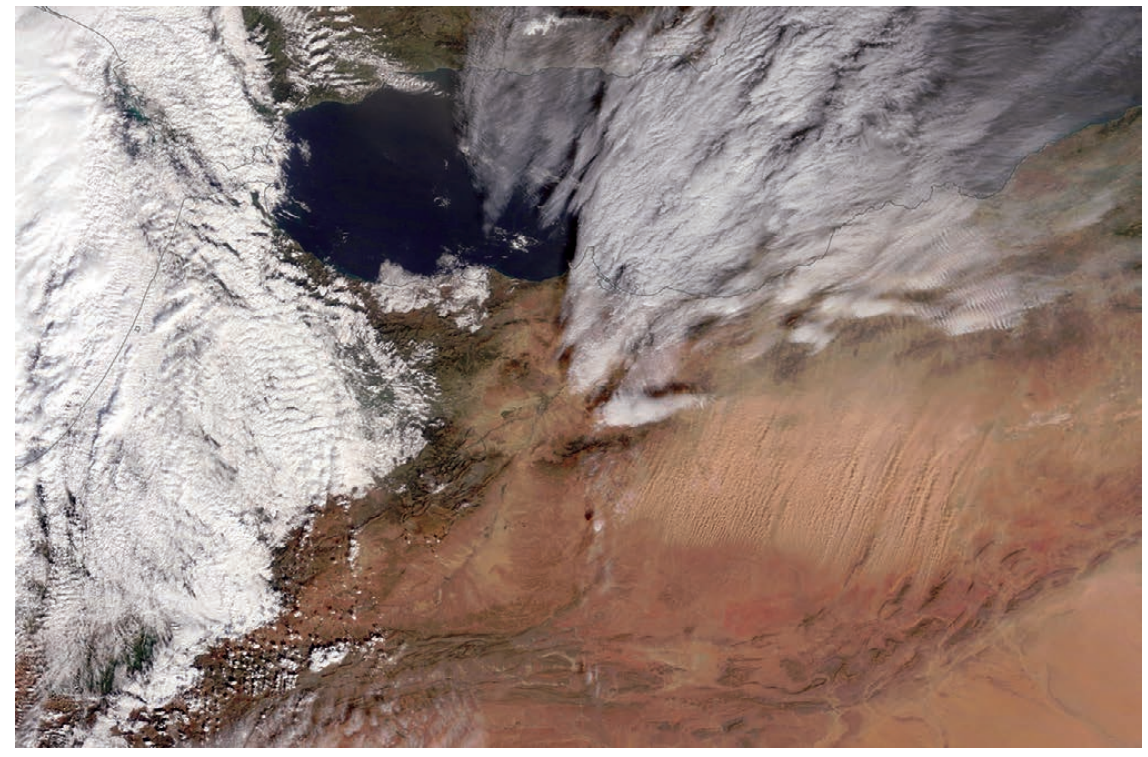

Figure 4. Image en composition colorée le 5 février 2021. Source : Nasa Worldview. 
L'absorption d'énergie est donc plus importante. D'ailleurs les poussières présentes dans le manteau neigeux sont capables d'absorber le rayonnement solaire jusqu'à $30 \mathrm{~cm}$ de profondeur (Latu et Goetz, 2016), faisant monter la température de la couche de neige avec poussières aux alentours de $0{ }^{\circ} \mathrm{C}$ et contribuant directement à la fonte de la neige. À noter que la concentration des poussières augmente au fil de la saison par la fonte printanière par le haut et leur caractère peu soluble
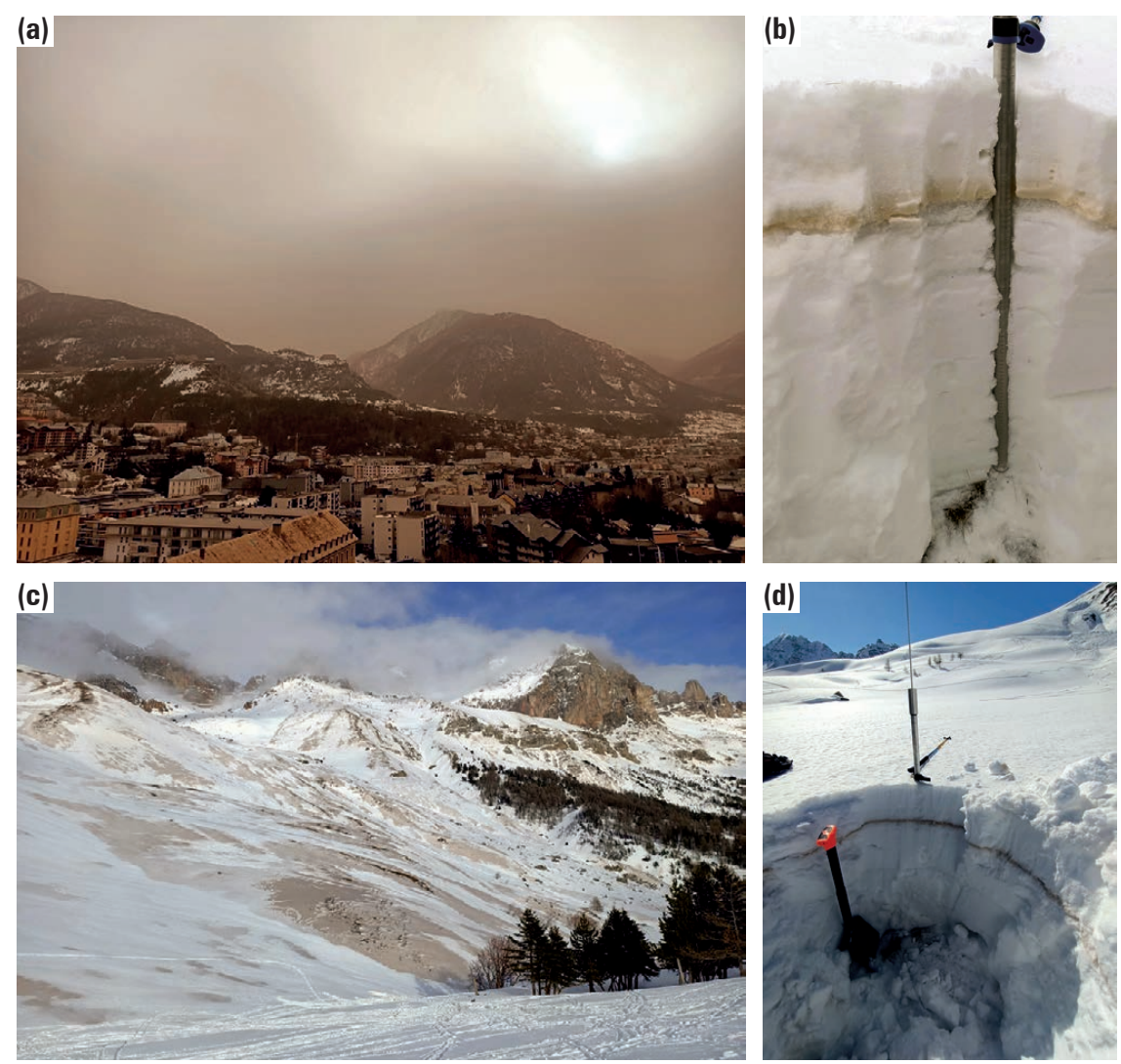

Figure 5. (a) Ambiance orangée à Briançon le 6 février ; (b) sondage de Montgenèvre le 12 février où la couche est à $10 \mathrm{~cm}$ de profondeur ; (c) col du Lautaret le 9 février ; (d) sondage de Vars le 25 février où la couche est à $18 \mathrm{~cm}$. Source : Gaétan Heymes.

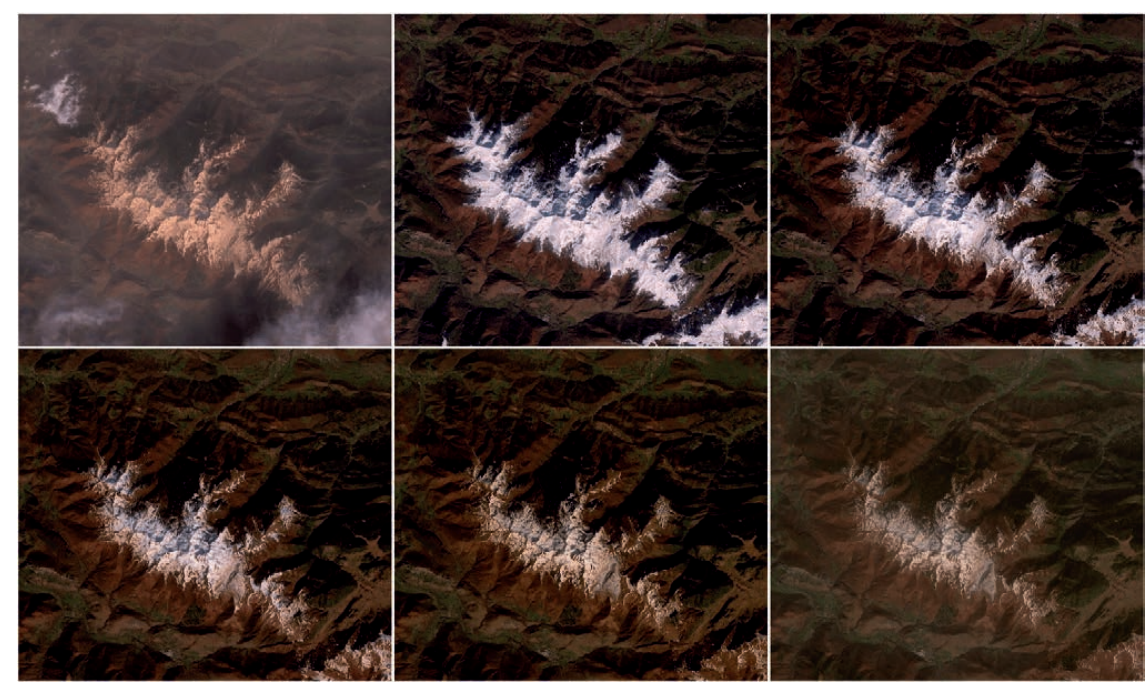

Figure 6. De gauche à droite et de haut en bas : évolution du manteau neigeux sur le massif de Tabe (Ariège) les $6,11,14,19,24$ février et $1^{\text {er }}$ mars. $1 \mathrm{~cm}$ représente environ $2 \mathrm{~km}$.. Source : Sentinel-2. étudiés dans les Alpes (Di Mauro et al., 2019). Concernant le Caucase, l'ordre de grandeur est sensiblement le même, avec 15 à 23 jours estimés (Dumont et al., 2020). Cette année, au fil des jours, le redoux va faire fondre la neige plus jeune pour faire réapparaître la couche ocre qui va persister jusqu'en fin de saison (là où elle n'est pas recouverte), ce qui va accélérer la fonte du manteau neigeux. Au fil des images (figure 6), la couche ocre réapparaît par l'extérieur des zones enneigées, donc à des altitudes moins élevées, avant de se généraliser.

\section{Situation des 2 et 3 mars 2021}

Début mars, un nouvel épisode concerne le sud, puis tout le pays. Les dépôts de poussières sont peu remarqués en montagne, mais les conséquences de l'épisode sont multiples. Du côté de la prévision météorologique, la nébulosité est systématiquement sous-estimée de la France à l'Espagne et l'ouest du bassin méditerranéen. Il s'agit d'une couche épaisse de nuages élevés, doublée quelquefois de nuages moyens. Dès le 2 mars, les basses valeurs présentes sur le proche atlantique pilotent un flux de sud sur l'Europe de l'Ouest, faisant remonter des poussières désertiques et des nuages sur l'Espagne et le sud de la France. Les modèles, qu'il s'agisse d'Arpège ou celui du CEPMMT, sous-estiment cette zone, qui est prévue bien moins compacte (figure 7).

Vingt-quatre heures après, la zone nuageuse remonte et recouvre toute la moitié sud-est de la France, s'étire sur l'est de l'Espagne et s'enroule sur l'Algérie. Les prévisions des modèles sont encore plus décevantes (figure 8). Alors que les nuages sont bien prévus sur l'Algérie, ils sont très mal anticipés sur l'Espagne et encore plus sur la France. Le modèle du CEPMMT est même très loin des observations. Ces erreurs de prévision de nébulosité rejaillissent sur les températures maximales, avec des prévisions qui surestiment les observations de 2 à $3{ }^{\circ} \mathrm{C}$, voire $4{ }^{\circ} \mathrm{C}$ localement, sur les régions concernées par ce vaste banc de cirrostratus et altostratus.

D'où viennent ces erreurs répétées par tous les réseaux de tous les modèles pendant plus de 48 heures ? Si l'on compare les profils verticaux simulés et les radiosondages au-dessus 
de Nîmes et de Majorque, les différences ne sont pas évidentes dans la mesure où les profils se rapprochent sur une forte humidité (souvent proche de la saturation) de $700 \mathrm{hPa}$ jusqu'à la tropopause vers $250 \mathrm{hPa}$ (figure 9). Il y a juste un léger écart en humidité entre les niveaux 700 et $600 \mathrm{hPa}$.

Si les modèles sont très proches en humidité, mais pas en nébulosité, cela ne peut qu'être lié à des processus relevant de la microphysique. Ici, la concentration en poussières désertiques est importante et ces poussières servent probablement de noyaux glaçogènes, entraînant donc de la nucléation dite hétérogène, puis la formation des nuages. Selon Sassen et al. (2003), dans un environnement identique, de la glaciation est intervenue pour former des altocumulus par des températures situées entre -5 et $-9{ }^{\circ} \mathrm{C}$, c'est-à-dire beaucoup moins froides qu'avec les noyaux glaçogènes usuels (dont la température d'activation se situe souvent entre -10 et $-14{ }^{\circ} \mathrm{C}$ ) et avec une sursaturation probablement moindre. Afin d'étayer cette thèse, il suffit de comparer les prévisions de concentration en aérosols issues de Mocage et les images satellitaires (figure 10). Qu'il s'agisse du 2 ou du 3 mars, la corrélation est parfaite entre les plus fortes concentrations et les nuages observés. Comme les schémas de microphysique des modèles n'utilisent que des paramétrisations génériques, ne considérant donc pas ces aérosols (nature et quantité), ils pourraient donc être à l'origine de cette nébulosité et des erreurs de prévision des modèles ces deux jours.

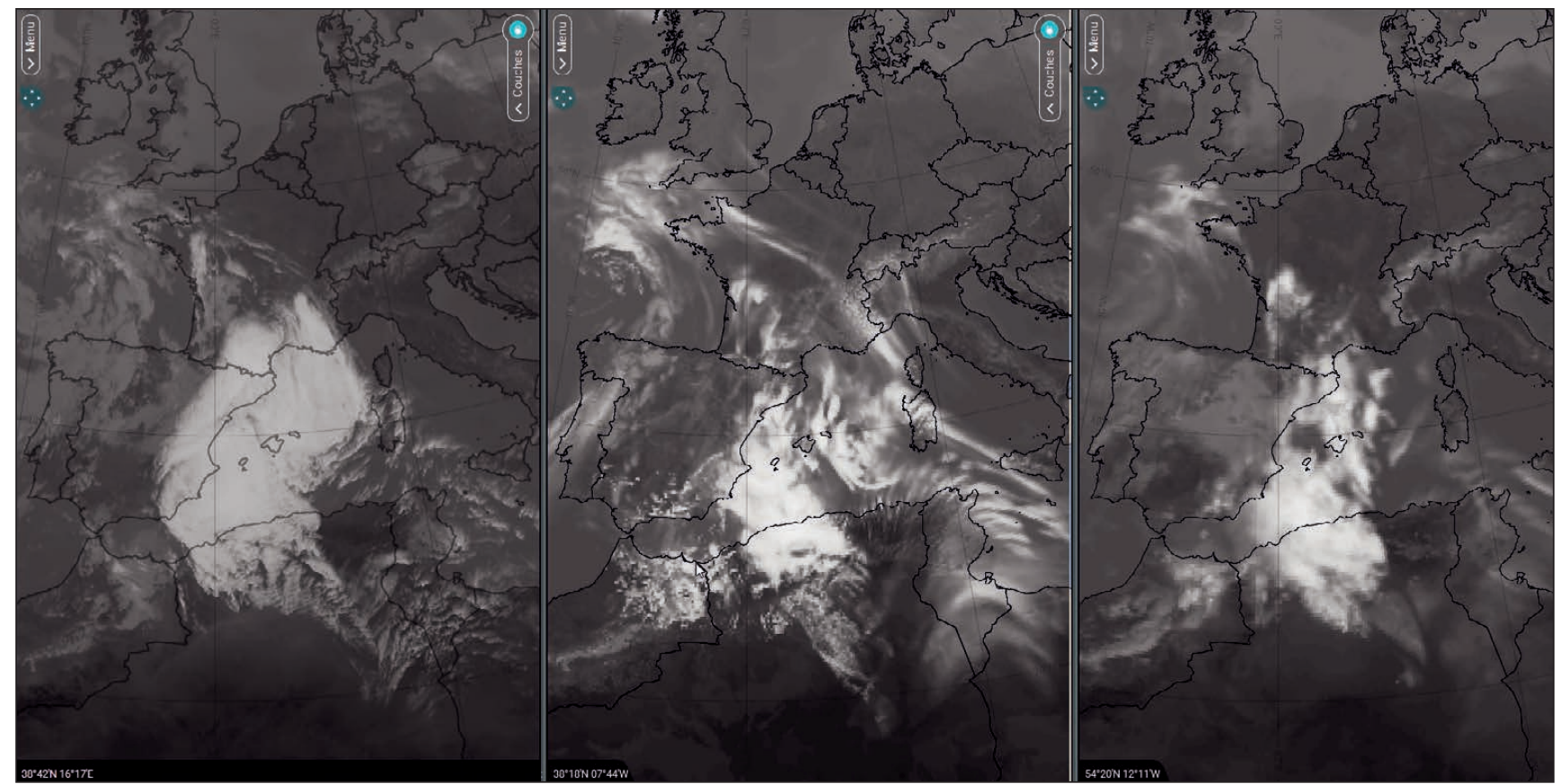

Figure 7. Image dans le canal infrarouge le 2 mars à 12 h TU, observée (à gauche), modélisée par Arpège (au centre) et le modèle du CEPMMT (à droite), modèles du 2 mars à $0 \mathrm{~h}$ TU. Source : Météo-France.

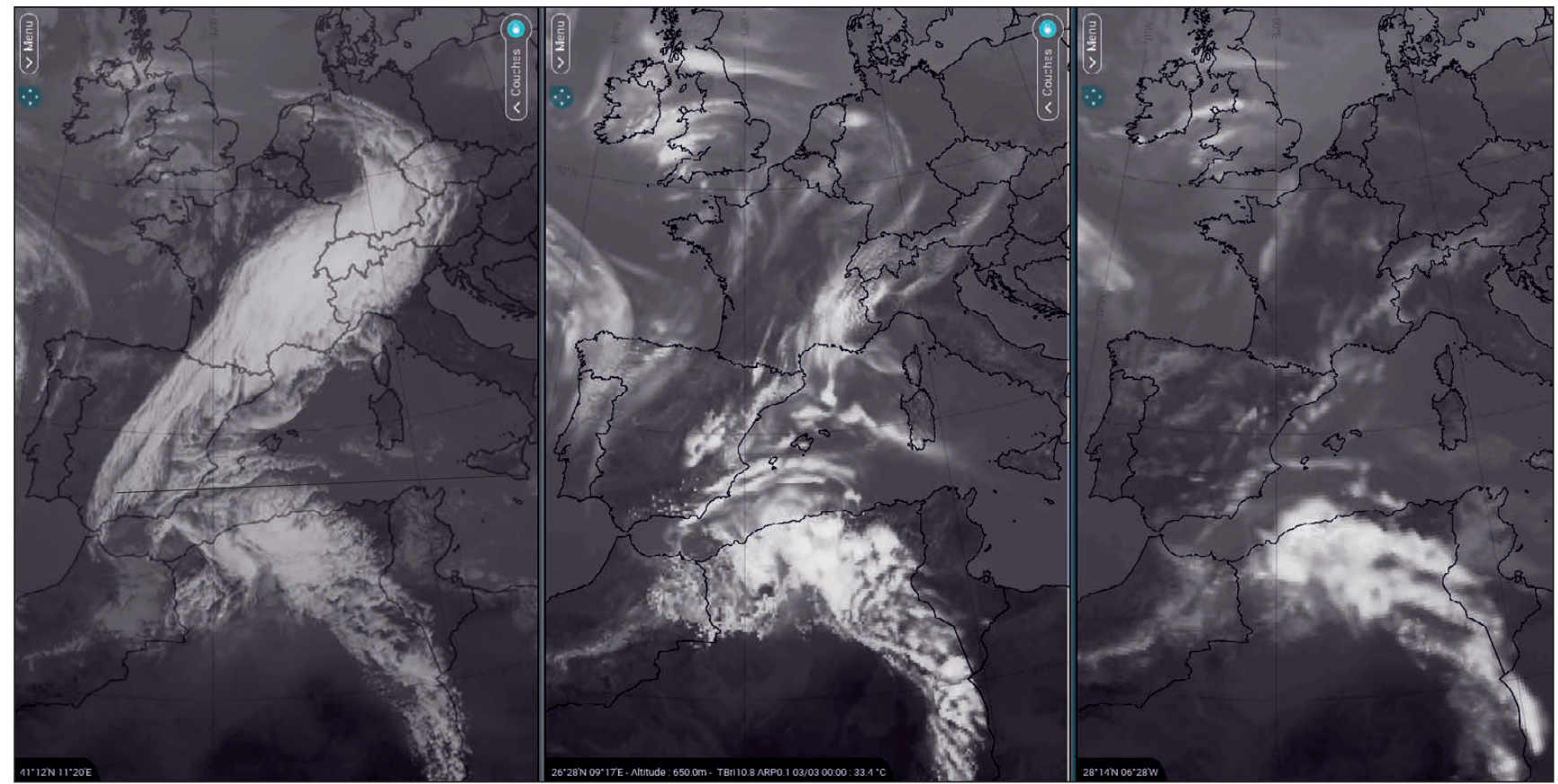

Figure 8. Image dans le canal infrarouge le 3 mars à 12 h TU, observée (à gauche), modélisée par Arpège (au centre) et le modèle du CEPMMT (à droite), modèles du 3 mars à 0 h TU. Source : Météo-France. 

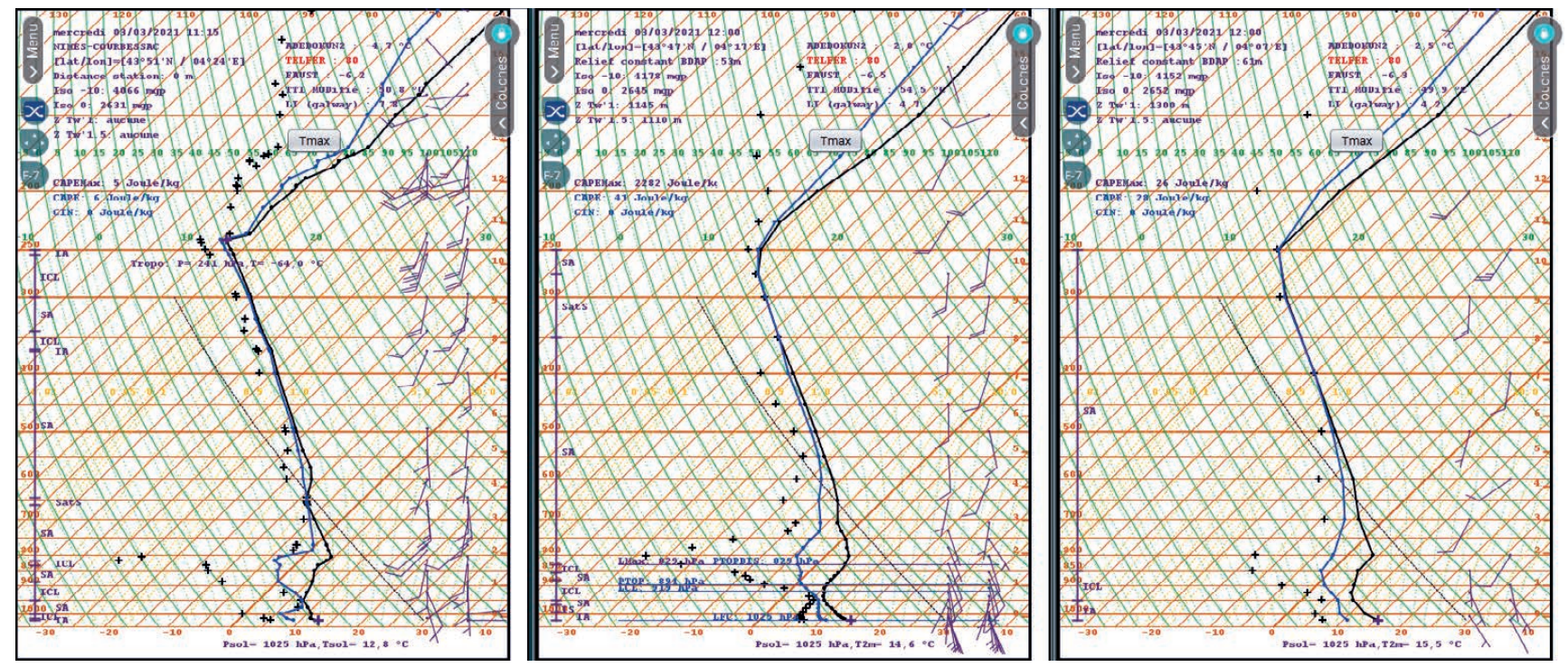

Figure 9. Comparaison entre le radiosondage de Nîmes (à gauche) et les profils verticaux simulés par Arpège (au centre) et du modèle du CEPMMT (à droite), le 3 mars à 12 h TU, modèles de 0 h TU. Source : Météo-France.
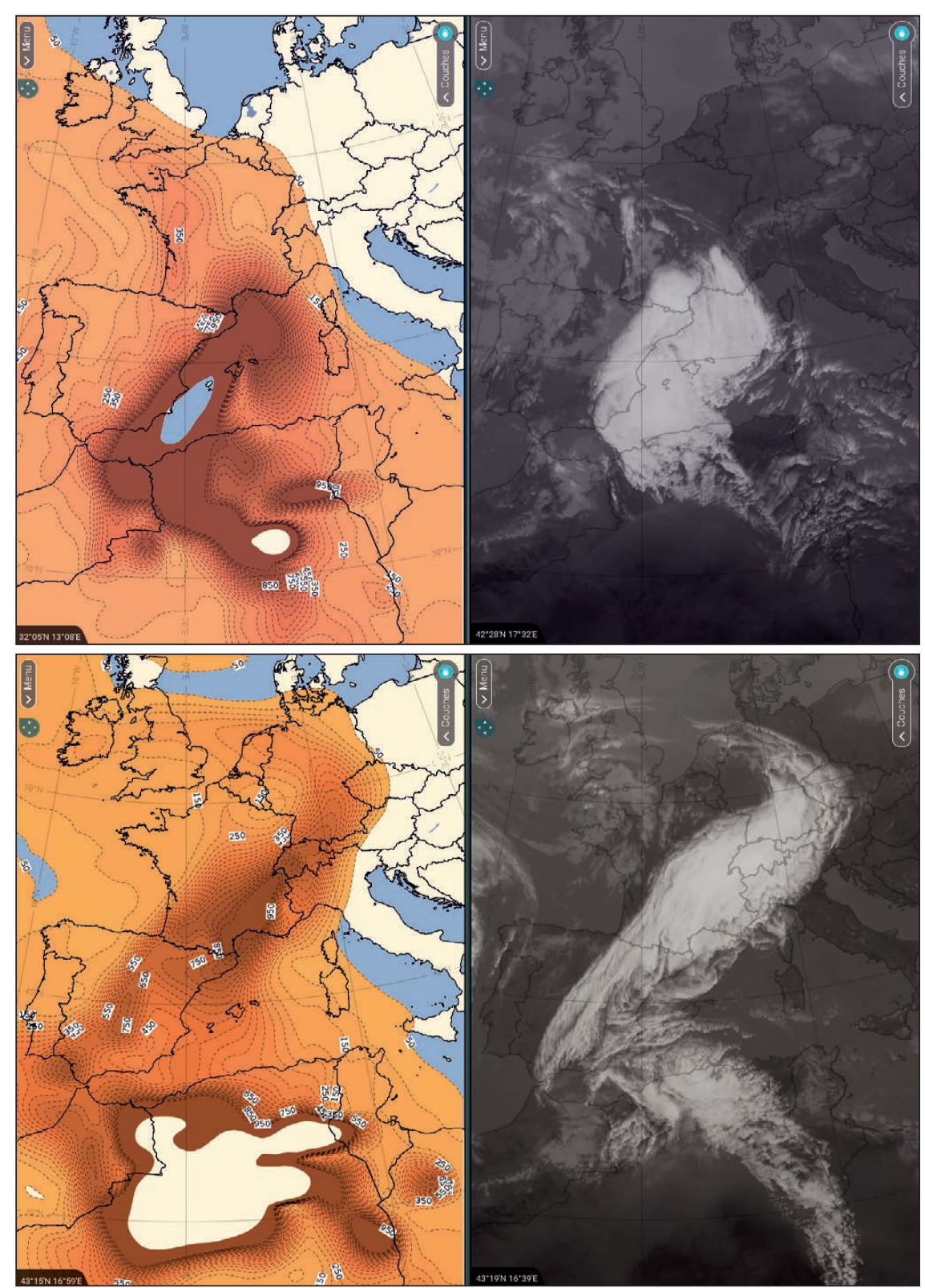

Figure 10. Comparaison entre l'image infrarouge (à droite) et la concentration en aérosols sur la colonne (à gauche) par le modèle Mocage pour le 2 mars à $12 \mathrm{~h}$ TU (groupe du haut) et le 3 mars à $12 \mathrm{~h}$ TU (groupe du bas), modèles de $0 \mathrm{~h}$ TU des jours concernés.
Les poussières transportées jusqu'en Europe sont majoritairement grossières, de taille comprise entre 2,5 et $10 \mu \mathrm{m}$ (note InVS; Di Mauro et al., 2019). Elles contribuent par exemple à hauteur de $45 \%$ de l'ensemble des PM10 dans le sud-est de l'Espagne et $35 \%$ à Chypre et sur les îles grecques (Pey et al., 2013). Néanmoins, les publications ne sont pas toutes en accord sur les conséquences sur la santé humaine. La plupart des publications proposées par l'InVS indiquent un risque significativement accru de problèmes de santé en période d'afflux de poussières désertiques. Cette analyse est toutefois à relativiser si l'on se réfère à Pandolfi et al. (2014). En effet, selon cette dernière étude, les phénomènes de vents de sable sont associés à des hauteurs de couches limites plus basses. Ainsi, au-delà des indicateurs en PM10 ou PM2.5, les polluants d'origine humaine sont plus concentrés dans cette couche limite et même si ce n'est pas démontré, peuvent être à l'origine de la hausse des pathologies humaines, devant les poussières désertiques. Néanmoins, l'InVS relève que le problème ne vient pas de la toxicité de ces particules, mais de l'obstruction partielle qu'elles peuvent entraîner dans l'appareil respiratoire. L'InVS complète : " Compte tenu des résultats des études épidémiologiques, certains auteurs recommandent que les épisodes de vents de sable, alors qu'ils sont inévitables, fassent l'objet de messages d'alerte sanitaire, particulièrement en direction des populations vulnérables. »

Début mars, en surface, la qualité de l'air se dégrade, en particulier en Occitanie, en devenant mauvaise à 
très mauvaise ${ }^{2}$. Le commentaire est éloquent : " Les concentrations en particules resteront élevées en raison de l'apport de particules désertiques qui viennent s'ajouter aux particules émises localement. ».

En montagne, les dépôts de poussière réchauffent et humidifient la neige : les avalanches de fonte sont plus précoces en journée ou inhabituelles pour la saison (Dumont et al., 2016). Il peut aussi y avoir des ruptures spontanées de plaques reposant sur cette couche de poussières par perte de cohésion par humidification. De plus, lorsque cette couche de neige orangée et à $0^{\circ} \mathrm{C}$ est recouverte par une couche de neige fraîche et froide, il peut y avoir un fort gradient vertical de température favorisant la métamorphose de la neige en des grains anguleux, diminuant la cohésion avec les couches supérieures.

Ce changement de stabilité du manteau neigeux en lien avec un dépôt de sable est très dépendant des conditions nivo-météorologiques immédiatement postérieures à ce dépôt (Chomette et al., 2017). Si de nouvelles chutes de neige se produisent très rapidement, la perte de cohésion est limitée.

Les épisodes de dépôt de poussières désertiques de début 2021 (essentiellement autour du 6 février) sont à l'origine de plusieurs avalanches. Le 20 mars 2021, en Haute Maurienne,

2. https://www.atmo-occitanie.org/ une avalanche se produit à $2600 \mathrm{~m}$ d'altitude sur un versant exposé nordest au col du Rocher Gris (figure 11). Il s'agit d'une plaque dure, avec départ linéaire. Ici la couche fragile persistante est juste en dessous des poussières bien visibles. Cela a été souvent remarqué sur le terrain avant le redoux de la fin mars, sur des versants froids. Les poussières se sont déposées sur tous les versants, puis ont eu lieu des épisodes neigeux de plus en plus froids, mais pas très abondants. Le fort gradient thermique a ainsi pu former une couche fragile persistante sous la couche de sable. Ici, la partie cohésive de la structure de plaque est composée de la neige récente du gros épisode de la mi-mars qui a pris de la cohésion par métamorphose de faible gradient.

Puis, le 30 mars 2021, une avalanche se produit dans le secteur du col des Marches (figure 12): avalanche de fonte, départ linéaire. Le manteau s'est progressivement tassé et humidifié (isotherme $0{ }^{\circ} \mathrm{C}$ à $3000 \mathrm{~m}$ depuis la veille), jusqu'à ce que l'équilibre mécanique soit rompu par l'humidification de la couche au-dessus des poussières, qui ne sont plus suffisamment enfouies. Probablement qu'en l'absence de poussières il n'y aurait pas eu cette avalanche ou elle se serait déclenchée plus tard, et pas forcément de manière linéaire. Cela montre qu'il y a encore un peu de cohésion, le manteau n'est pas totalement « pourri » et donc les habitués peuvent être surpris.

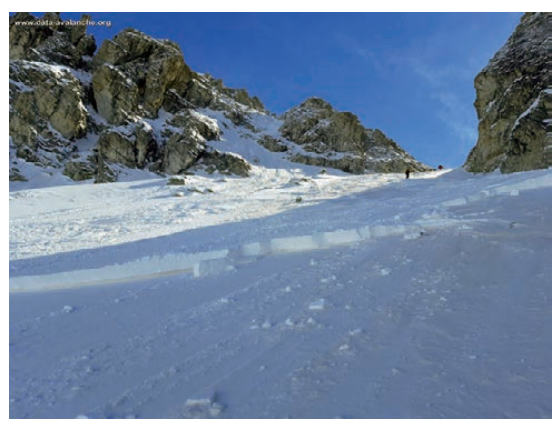

Figure 11. Avalanche au col du Rocher Gris le 20 mars 2021. Source : https://data-avalanche.org.

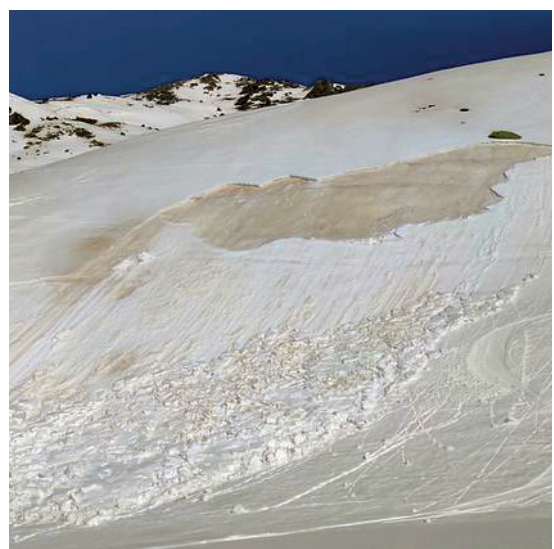

Figure 12. Avalanche au col des Marches le 30 mars 2021. Source : https://data-avalanche.org.

\section{Remerciements}

Merci à Simon Gascoin (Cesbio) pour la fourniture des images issues du satellite Sentinel-2. Merci également à Romain Dieumegard et Didier Bonfils pour les photographies des avalanches déposées sur https://data-avalanche.org.

\section{Bibliographie}

Chomette L., Tuzet F., Dumont M., 2017. La neige et le sable font-ils bon ménage ? Neige et Avalanches, 159, 8-12.

Cottereau J., 2020. Les brumes de poussières sahariennes aux Antilles françaises et en Guyane. La Météorologie, 110, 34-45. doi: 10.37053/lameteorologie-2020-0068

Di Mauro B., Garzonio R., Rossini M., Filippa G., Pogliotti P., Galvagno M., Morra di Cella U., Migliavacca M., Baccolo G., Clemenza M., Delmonte B., Maggi V., Dumont M., Tuzet F., Lafaysse M., Morin S., Cremonese E., Colombo R., 2019. Saharan dust events in the European Alps: role in snowmelt and geochemical characterization. The Cryosphere, 13, 1147-1165. doi: 10.5194/tc-13-1147-2019

Dumont M., Dufour A., Flin F., Hagenmuller P., Lejeune Y., 2016. Effet du dépôt de sable dans le manteau neigeux. Note de travail interne à Météo-France.

Dumont M., Tuzet F., Gascoin S., Picard G., Kutuzov S., Lafaysse M., Cluzet B., Nheili R., Painter T.H., 2020. Accelerated snow melt in the Russian Caucasus mountains after the Saharan dust outbreak in March 2018. J. Geophys. Res.: Earth Surface, 125, e2020JF005641. doi: 10.1029/2020JF005641

Latu V., Goetz D., 2016. Les neiges colorées. Neige et Avalanches, 154, 27-28.

Pandolfi M., Tobias A., Alastuey A., Sunyer J., Schwartz J., Lorente J., Pey J., Querol X., 2014. Effect of atmospheric mixing layer depth variations on urban air quality and daily mortality during Saharan dust outbreaks. Sci. Total. Environ., 494-495, 283-289. doi: 10.1016/j.scitotenv.2014.07.004

Pey J., Querol X., Alastuey A., Forastiere F., Stafoggia M., 2013. African dust outbreaks over the Mediterranean Basin during 2001-2011: PM concentrations, phenomenology and trends, and its relation with synoptic and mesoscale meteorology. Atmos. Chem. Phys., 13, 1395-1410. doi: 10.5194/acp-13-1395-2013

Sassen K., DeMott P.J., Prospero J.M., Poellot M.R., 2003. Saharan dust storms and indirect aerosol effects on clouds: CRYSTAL-FACE results. Geophys. Res. Lett., 30, 12, 35-1 35-4. doi: 10.1029/2003GL017371

Tuzet F., Dumont M., Lafaysse M., Picard G., Arnaud L., Voisin D., Lejeune Y., Charrois L., Nabat P., Morin S., 2017. A multilayer physically based snowpack model simulating direct and indirect radiative impacts of light-absorbing impurities in snow. The Cryosphere, 11, 2633-2653. doi: 10.5194/tc-11-2633-2017 